\title{
Triptonide inhibits metastasis potential of thyroid cancer cells via astrocyte elevated gene-1
}

\author{
Liangjie Fu\#, Xiaohong Niu", Ruhui Jin, Feiyun Xu, Jiguo Ding, Li Zhang, Zihui Huang \\ Department of Scrofulosis, Nanjing Integrated Traditional Chinese and Western Medicine Hospital, Nanjing 210014, China \\ Contributions: (I) Conception and design: L Fu, F Xu; (II) Administrative support: R Jin, X Niu; (III) Provision of study materials or patients: J Ding, \\ R Jin; (IV) Collection and assembly of data: L Zhang, X Niu; (V) Data analysis and interpretation: Z Huang, L Fu; (VI) Manuscript writing: All \\ authors; (VII) Final approval of manuscript: All authors. \\ "These authors contributed equally to this work. \\ Correspondence to: Zihui Huang. Department of Scrofulosis, Nanjing Integrated Traditional Chinese and Western Medicine Hospital, No.179, \\ Xiaolingwei, Xuanwu District, Nanjing 210014, China. Email: huangzih_hzhui@163.com.
}

\begin{abstract}
Background: Triptonide (TN) was recently proved to have anti-tumor effects. The current study explored whether TN inhibited thyroid cancer and the possible underlying mechanism.

Methods: MDA-T68 and BCPAP cells were treated by TN. Cell viability, migration and invasion rate were detected by MTT and Transwell. Protein expressions were determined by Western blot and mRNA expressions were detected by Real-time Quantitative PCR (qPCR).

Results: TN at the concentration higher than $50 \mathrm{nmol} / \mathrm{L}$ inhibited cell viability, migration and invasion of MDA-T68 and BCPAP cells, and astrocyte elevated gene (AEG-1) expression, was decreased by TN at the concentration higher than $50 \mathrm{nmol} / \mathrm{L}$. Furthermore, $A E G-1$ overexpression inhibited cell viability, migration and invasion capacity of MDA-T68 and BCPAP cells, while TN reduced AEG-1 expression, and weaken the effect of $A E G-1$ overexpression on cell viability, migration and invasion capacities. Moreover, TN depressed the increase of matrix metalloproteinase (MMP) 2, MMP9 and N-cadherin expressions caused by AEG-1 overexpression. Meanwhile, E-cadherin expression reduced by AEG-1 overexpression was increased by TN. Conclusions: TN could inhibit the metastasis potential of thyroid cancer cells through inhibiting the expression of AEG-1. Our findings reveal the mechanism of TN in the treatment of thyroid cancer, which should be further explored in the study of thyroid cancer.
\end{abstract}

Keywords: Triptonide; metastasis; thyroid cancer; regulation; drug monomer

Submitted Aug 29, 2019. Accepted for publication Nov 29, 2019.

doi: $10.21037 /$ tcr.2019.12.94

View this article at: http://dx.doi.org/10.21037/tcr.2019.12.94

\section{Introduction}

Thyroid cancer is the most common endocrine system malignancy, accounting for about $1 \%$ systemic malignancies (1). Papillary, follicular, undifferentiated and medullary are four major pathological phenotypes of thyroid cancer (1). Traditional treatment strategies are mainly surgical resection and radioactive iodine treatment, but there will be complications that seriously affect the quality of life of patients, such as parathyroid glands, recurrent laryngeal nerves and laryngeal nerve injury (2). In addition, undifferentiated thyroid cancer accounts for only $2 \%$ thyroid cancer, but we still lack effective approaches to control and inhibit its progression, due to local invasion, distant metastasis of thyroid cancer cells (3). Therefore, the search for new therapeutic drugs is imminent.

Tripterygium wilfordii is a traditional Chinese medicine with anti-inflammatory, anti-tumor and immunosuppressive effects, but its efficacy was reduced by its toxicity (4). 
At present, from Tripterygium wilfordii, multiple active components have been successfully extracted, including Triptonide (TN) (5). Previous study showed that TN did not cause toxic effects on mice treated by 20 -fold effective dose of TN (5). However, increasing evidences indicate that $\mathrm{TN}$ can act potentially as an anti-cancer drug for treatment of cancers $(6,7)$. Nevertheless, studies were less conducted on the anti-tumor effects of TN on thyroid cancer, and its anti-tumor activity has not been fully explored.

The current study aimed to explore whether TN could inhibit thyroid cancer and the possible mechanism. The current findings provide a novel pathway to intervene the process of thyroid cancer in addition to invasive approaches.

\section{Methods}

\section{Cell culture}

MDA-T68 cell line was purchased from American Type Culture Collection, while BCPAP was obtained from German Collection of Microorganisms and Cell Cultures $\mathrm{GmbH}$. For cell culture, the cells were cultured in Roswell Park Memorial Institute (RPMI)1640 (21875091, Thermo Fisher, Waltham, USA) containing 10\% fetal bovine serum (FBS, F8192, Sigma-Aldrich, Missouri, USA) with $5 \% \mathrm{CO}_{2}$ at $37^{\circ} \mathrm{C}$.

\section{Protocol}

MDA-T68 and BCPAP cells were divided into Control, DMSO (Dimethyl sulfoxide), TN groups. TN (CAS: 38647-11-9, $\mathrm{C}_{20} \mathrm{H}_{22} \mathrm{O}_{6}$, MW: 358.39, Shanghai Linzyme Biosciences Ltd.) powder was dissolved in DMSO at a final concentration of $5 \mathrm{mmol} / \mathrm{L}$ (D2650, Sigma-Aldrich, Missouri, USA), and further diluted to concentrations of to 10,50 and $100 \mathrm{nmol} / \mathrm{L}$. Briefly, MDA-T68 and BCPAP cells in DMSO group was treated by $1 \%$ DMSO mixed in culture medium. The cells in TN groups were treated by 10, 50 and $100 \mathrm{nmol} / \mathrm{L}$ TN for $24 \mathrm{~h} \mathrm{(8).} \mathrm{For}$ the exploration of TN effects on astrocyte elevation gene1 (AEG-1), MDA-T68 and BCPAP cells were divided into Control, negative (NC), AEG-1, AEG-1 + TN and TN groups. The cells in NC group were transfected with pcDNA3.1 (+) plasmid (GenePharma, Shanghai, China), while those in AEG-1 groups were transfected by AEG1 overexpression plasmid (GenePharma, Shanghai, China) using Lipofectamine 3000 (L3000015, Thermo Fisher, Waltham, USA). Based on the treatment aforementioned, the cells in AEG-1 + TN group were transfected with AEG1 overexpression plasmid and further treated by $50 \mathrm{nmol} / \mathrm{L}$ $\mathrm{TN}$ (according to the results in the present study). The cells in TN group were only treated by $50 \mathrm{nmol} / \mathrm{L} \mathrm{TN}$. All cells in Control group were cultured in medium only.

\section{MTT (3-(4,5-Dimethylthiazol-2-yl)-2,5- Diphenyltetrazolium Bromide)}

Proliferation of MDA-T68 and BCPAP cells were determined by Cell Proliferation Kit I (11465007001, Sigma-Aldrich, Missouri, USA). Briefly, when cells reached logarithmic phase, the cells were diluted to $5 \times 10^{6}$ cells $/ \mathrm{mL}$ and then planted in a plate. $40 \mu \mathrm{L}$ MTT formulated in serum-free media was further added to the cells. MTT was dissolved by MTT Solvent for $15 \mathrm{~min}$ after culturing in MTT solution at $37^{\circ} \mathrm{C}$ for $3 \mathrm{~h}$. A microplate reader (Multiskan, Thermo, Waltham, USA) was used for the detection of optic density (OD) at $590 \mathrm{~nm}$.

\section{Transwell assay}

Migration and invasion rates of MDA-T68 and BCPAP cells were determined by Transwell $(8-\mu \mathrm{m}, \mathrm{BD}$ Biosciences, CA, USA). For invasion assay, $1 \times 10^{5}$ cells were resuspended and seeded into the upper chamber of Transwell pre-coated by Matrigel (BD Bioscience, CA, USA) in the middle of upper and bottom chambers. A mixture of $500 \mu \mathrm{L}$ medium supplied with $10 \%$ FBS were pipetted into the lower chamber. The chambers were incubated with $5 \% \mathrm{CO}_{2}$ at $37{ }^{\circ} \mathrm{C}$ for $24 \mathrm{~h}$. After washing, the cells were fixed by $4 \%$ paraformaldehyde and $0.2 \%$ crystal violet for 15 and $8 \mathrm{~min}$, respectively, and photographed under a microscope. For migration assay, similar process was performed as invasion assay but without Matrigel-coated membrane.

\section{Western blot}

Proteins in MDA-T68 and BCPAP cells were extracted by the mixture of Radio Immunoprecipitation Assay (RIPA) lysate (R0278, Sigma-Aldrich, Missouri, USA) and protease inhibitor (S8830, Sigma-Aldrich, Missouri, USA). After the extraction, the concentration of total protein was determined by bicinchoninic acid (BCA) method (BCA1, Sigma-Aldrich, Missouri, USA). According to the results of BCA, protein sample in each group was separated on $10 \%$ sodium dodecyl sulfate polyacrylamide gel electrophoresi 
Table 1 primers used in the present study

\begin{tabular}{ll}
\hline Primer name & Sequence (5'-3') \\
\hline AEG-1 forward & GAATCTCCGGAGCGAGGAAC \\
AEG-1 reverse & GGTGGCTGCTTTGCTGTTAC \\
MMP2 forward & AGCGAGTGGATGCCGCCTTTAA \\
MMP2 reverse & CATTCCAGGCATCTGCGATGAG \\
MMP9 forward & GCCACTACTGTGCCTTTGAGTC \\
MMP9 reverse & CCCTCAGAGAATCGCCAGTACT \\
E-cadherin forward & GCCTCCTGAAAAGAGAGTGGAAG \\
E-cadherin reverse & GCCTCCTGAAAAGAGAGTGGAAG \\
N-cadherin forward & CCTCCAGAGTTTACTGCCATGAC \\
N-cadherin reverse & GTAGGATCTCCGCCACTGATTC \\
$\beta$-actin forward & TACCTGAACCCGTGTTGCTCTC \\
$\beta$-actin reverse & GTTGCTGAGGTATCGCCAGGAA \\
\hline
\end{tabular}

(SDS-PAGE) at $100 \mathrm{~V}$ for $1.5 \mathrm{~h}$, and transferred to polyvinylidene difluoride (PVDF) membrane. After blocked by $5 \%$ milk (non-fat), the PVDF membrane was incubated with AEG-1 (H00092140-P01, Abnova, 27 KD), matrix metalloproteinase (MMP) 9 (ab73734, Abcam, San Francisco, USA, 78 KD), MMP2 (ab37150, Abcam, San Francisco, USA, 72 KD), E-cadherin (ab40772, Abcam, San Francisco, USA, 97 KD), N-cadherin (ab18203, Abcam, San Francisco, USA, $130 \mathrm{KD}$ ), beta-actin (ab8226, Abcam, San Francisco, USA, 42KD) at 1:1000 dilution at $4{ }^{\circ} \mathrm{C}$ overnight. Goat anti-rabbit secondary antibody (1:2000, ab205718, Abcam, San Francisco, USA) was used for further detection of primary antibody. Signals were recorded by exposing the protein bolts to enhanced chemiluminescence (ECL) (\#6883, SignalFire ${ }^{\mathrm{TM}}$ ECL Reagent).

\section{Quantitative polymerase chain reaction (qPCR)}

Total RNAs in MDA-T68 and BCPAP cells were extracted by TRIzol reagent (15596018, Thermo Fisher, Waltham, USA) and then reverse-transcribed into cDNAs using PrimeScript RT reagent kit (Takara Biotechnology Co., Ltd., Dalian, China). $2 \mu \mathrm{L}$ cDNA template, $12 \mu \mathrm{L} 2 \times$ SYBER Green master mix, $1 \mu \mathrm{L}$ forward primer $(10 \mu \mathrm{M})$, reverse primer $1 \mu \mathrm{L}(10 \mu \mathrm{M})$, and $9 \mu \mathrm{L}$ double distilled water $\left(\mathrm{ddH}_{2} \mathrm{O}\right)$ was prepared for qPCR FastStart Universal SYBR Green Master (Rox, 4913850001, Roche, Shanghai, China) in Bio-Rad IQ5 thermocycler (Bio-Rad, CA, USA), and the experiments were performed under the following conditions: at $95^{\circ} \mathrm{C}$ for $2 \mathrm{~min}, 40$ cycles at $95^{\circ} \mathrm{C}$ for $30 \mathrm{sec}$ and at $65{ }^{\circ} \mathrm{C}$ for $30 \mathrm{sec} .2^{-\Delta \Delta C t}$ method was used to calculate the mRNA levels of target genes. The primer sequences used in the present study were listed in Table 1.

\section{Statistical analysis}

The data collected from the present study were analyzed in Graphpad prism 8.0. Multiple comparisons between groups were conducted by One-way Analysis of Variance (ANOVA). Statistical significance was set as $\mathrm{P}<0.05$.

\section{Results}

\section{TN inbibited cell viability, migration and invasion of thyroid cancer cells}

To observe whether TN affected the proliferation and metastasis of thyroid cancer cells, we measured the cell viability, migration and invasion of MDA-T68 and BCPAP cells treated by $\mathrm{TN}$. We found that the relative cell viability, migration and invasion rates of MDA-T68 and BCPAP cells were suppressed by $\mathrm{TN}$ at the concentrations of 50 and $100 \mathrm{nmol} / \mathrm{L}$ as compared with Control group (Figure 1, $\left.{ }^{*} \mathrm{P}<0.05,{ }^{* *} \mathrm{P}<0.001\right)$. TN at $10 \mathrm{nmol} / \mathrm{L}$ significantly reduced relative migration rate of $\mathrm{BCPAP}$ cells (Figure $1 \mathrm{E},{ }^{*} \mathrm{P}<0.05$, indicating that $\mathrm{TN}$ could decrease tumorigenesis of the two cells.

\section{TN reduced AEG-1 expression in thyroid cancer cells}

To determine whether TN exerted its function in thyroid cancer cells through AEG-1, we measured AEG-1 expression in MDA-T68 and BCPAP cells treated by different concentrations of TN (10, 50, $100 \mathrm{nmol} / \mathrm{L})$. The results that AEG-1 expression was significantly reduced in MDA-T68 and BCPAP cells when TN concentrations were at 50 and $100 \mathrm{nmol} / \mathrm{L}$ (Figure $2,{ }^{* *} \mathrm{P}<0.001$ ), suggesting that $\mathrm{TN}$ could regulate the expression of AEG-1 and therefore exert its antitumor effect.

\section{TN partially eliminated the carcinogenesis of AEG-1 overexpression on cell viability, migration and invasion of thyroid cancer cells}

To investigate the relationship of TN and AEG-1 expression in the carcinogenesis of thyroid cancer cells, 
A
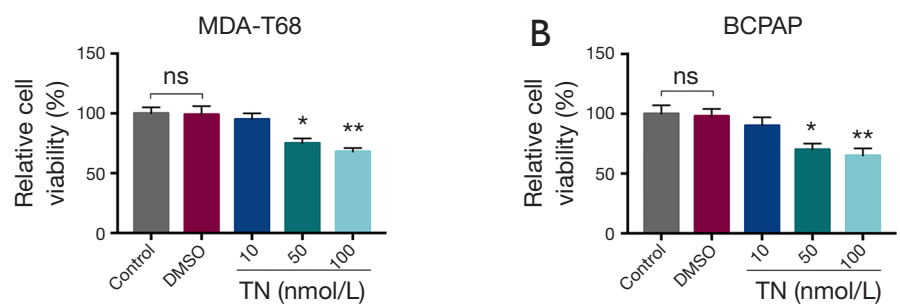

C

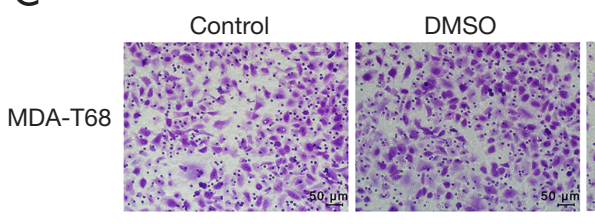
$\mathrm{TN}(\mathrm{nmol} / \mathrm{L})$
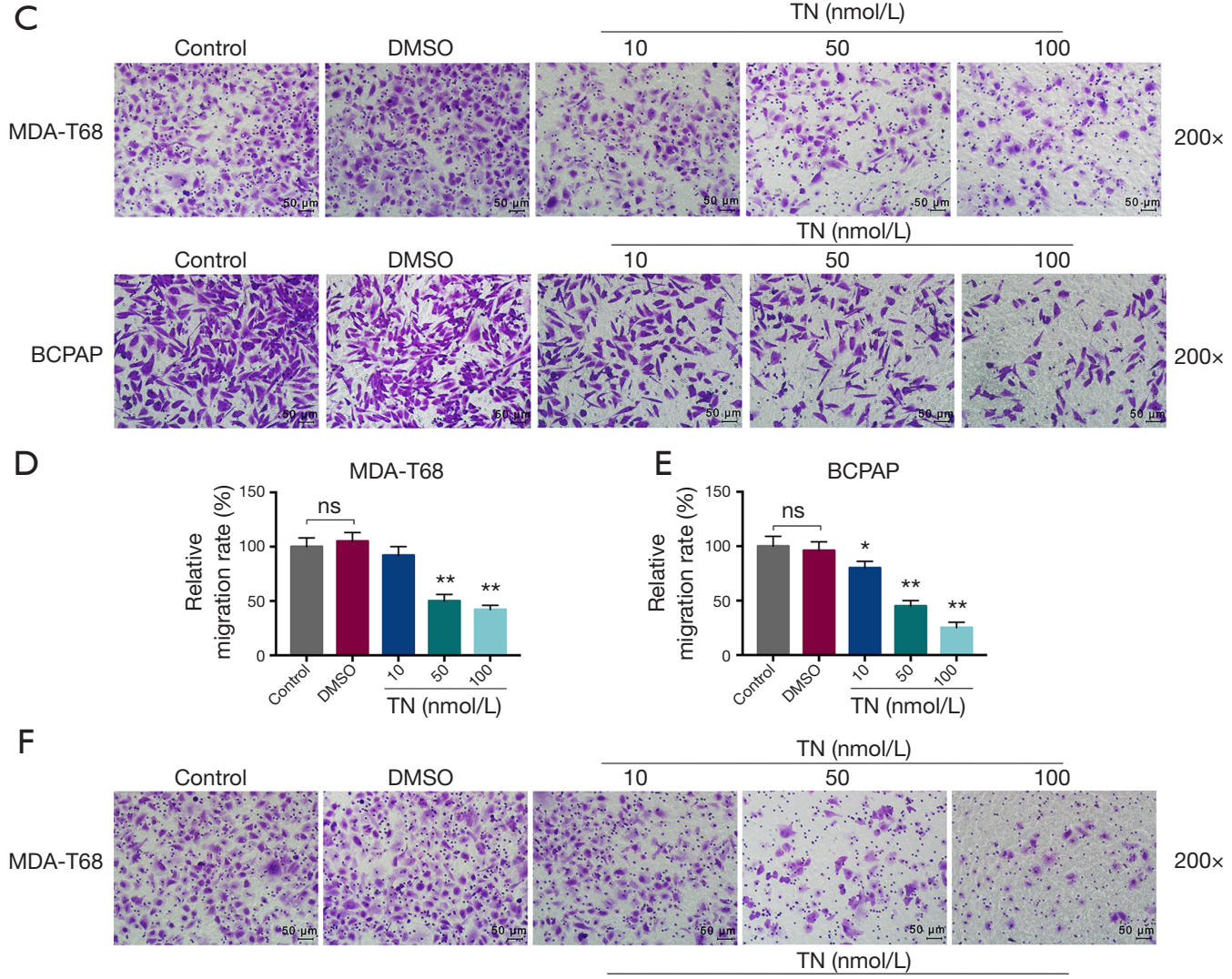

$200 \times$

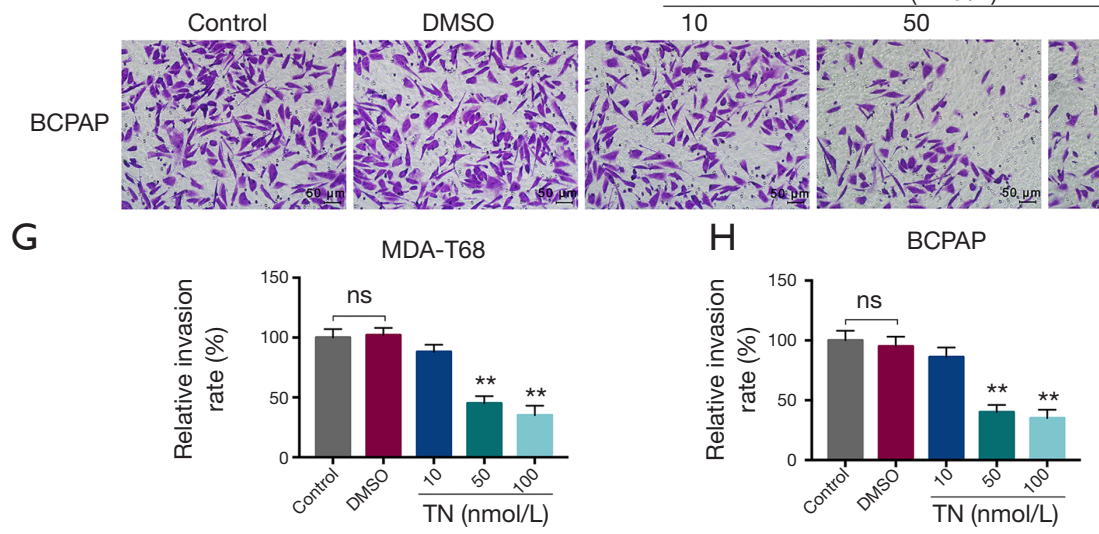

Figure 1 The effects of triptonide (TN) on the metastasis of thyroid cancer cells. (A) Relative cell viability in MDA-T68 and (B) BCPAP cells in Control, DMSO, $10 \mathrm{nmol} / \mathrm{L}, 50 \mathrm{nmol} / \mathrm{L}$, and $100 \mathrm{nmol} / \mathrm{L}$ TN groups; (C) representative images showed the MDA-T68 and BCPAP cells migrated into the bottom chamber in the groups, the cells were stained by $0.2 \%$ crystal violet; (D) relative migration rate (\%) in MDA-T68 and (E) BCPAP cells in the groups; (F) representative images showed the MDA-T68 and BCPAP cells invaded into the bottom chamber in the groups, the cells were stained by $0.2 \%$ crystal violet; $(\mathrm{G})$ relative invasion rate $(\%)$ in MDA-T68 and $(\mathrm{H})$ BCPAP cells in the groups. Bars indicated mean \pm standard deviation (SD). ${ }^{*} \mathrm{P}<0.05$ or ${ }^{* *} \mathrm{P}<0.001$ versus DMSO group. 
A

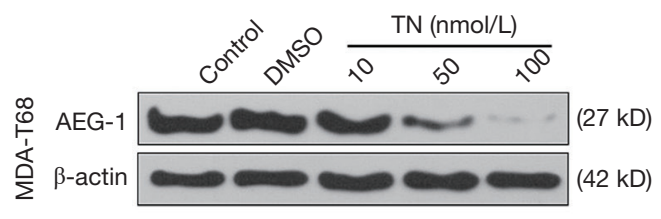

D

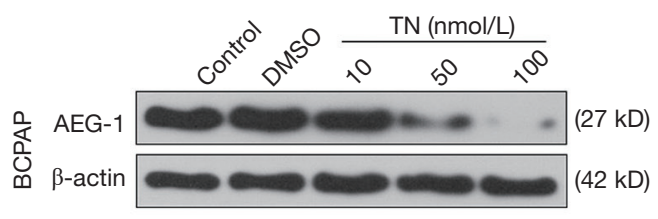

B

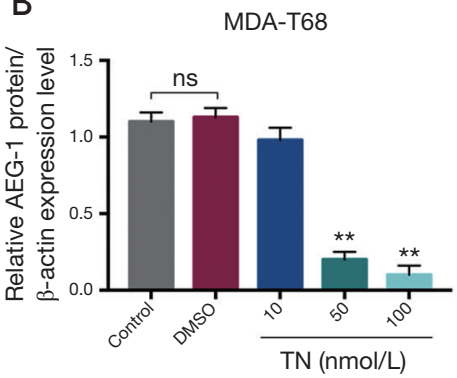

E

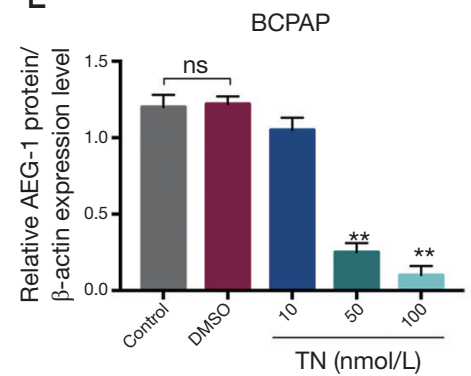

C

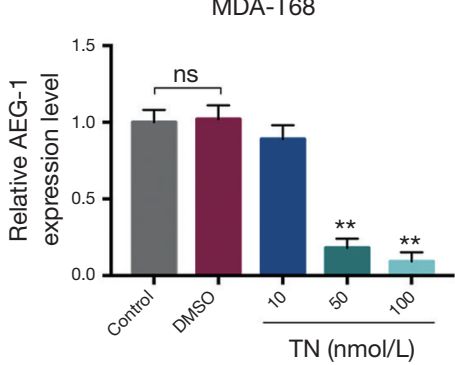

$\mathrm{F}$

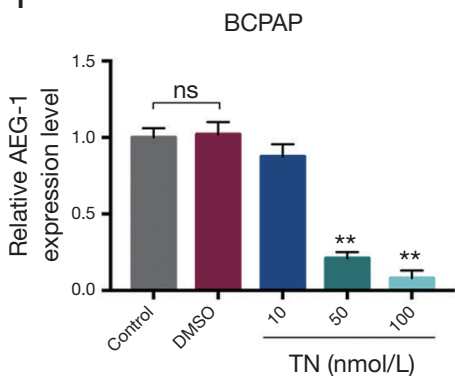

Figure 2 The effects of TN on astrocyte elevated gene (AEG)-1 expression in thyroid cancer cells. (A) AEG-1 protein bolts from MDA-T68 cells by Western blot in Control, DMSO, 10, 50 and $100 \mathrm{nmol} / \mathrm{L}$ TN groups; (B) relative AEG-1 protein expression and (C) mRNA expression in MDA-T68 cells in the groups; (D) AEG-1 protein bolts from BCPAP cells by Western blot in the groups; (E) relative AEG-1 protein expression and $(\mathrm{F}) \mathrm{mRNA}$ expression in BCPAP cells in the groups; Bars indicated mean $\pm \mathrm{SD}$. ${ }^{* *} \mathrm{P}<0.001$ versus DMSO group.

we observed the changes of metastasis of MDA-T68 and BCPAP cells with overexpressed AEG-1 after TN treatment. The data revealed that the relative cell viability, migration and invasion rates of MDA-T68 and BCPAP cells were elevated in AEG-1 group compared with NC group, while they were significantly lowered in AEG-1+TN group compared with AEG-1 group (Figure $3,{ }^{*} \mathrm{P}<0.05,{ }^{*} \mathrm{P}<0.001$; $\left.{ }^{\# \#} \mathrm{P}<0.001\right)$. In addition, the relative cell viability, migration and invasion rates of MDA-T68 and BCPAP cells in TN group was greatly lower than in AEG-1+TN group and Control group (Figure 3, ${ }^{* *} \mathrm{P}<0.001 ;{ }^{\wedge} \mathrm{P}<0.001$ ).

\section{TN inbibited epithelial-mesenchymal transition (EMT) process of thyroid cancer cells through down-regulating AEG-1 expression}

To explore whether TN regulated EMT process through modulating AEG-1, we determined the expression levels of AEG-1, MMP2, MMP9, E-cadherin and N-cadherin in MDA-T68 and BCPAP cells with overexpressed AEG1 after the treatment of TN. The results revealed the expressions of AEG-1, MMP2, MMP9 and N-cadherin of both MDA-T68 and BCPAP cells in AEG-1 group were lower than that in $\mathrm{NC}$ group (Figure $4,{ }^{*} \mathrm{P}<0.05$, ${ }^{* *} \mathrm{P}<0.001$ ), moreover, their expressions in AEG-1 + TN group were lower than that in AEG-1 group, but higher than that in TN group (Figure $4,{ }^{*} \mathrm{P}<0.05,{ }^{\# \#} \mathrm{P}<0.001$; $\left.{ }^{\wedge} \mathrm{P}<0.05,{ }^{\wedge} \mathrm{P}<0.001\right)$. Furthermore, the expressions of these genes of MDA-T68 and BCPAP cells in TN group were reduced in TN group compared with Control group (Figure 4 , $\left.{ }^{*} \mathrm{P}<0.05,{ }^{* *} \mathrm{P}<0.001\right)$. In addition, the expression of E-cadherin exhibited the opposite trend to the expressions of AEG-1, MMP2, MMP9 and N-cadherin (Figure 4, $\left.{ }^{*} \mathrm{P}<0.05,{ }^{* *} \mathrm{P}<0.001,{ }^{\#} \mathrm{P}<0.05 ;{ }^{\wedge \wedge} \mathrm{P}<0.001\right)$. Thus, the findings showed that AEG-1 might be involved in the effect of TN on inhibiting the EMT of thyroid cancer cells.

\section{Discussion}

The current study found that TN could decrease the proliferation, migration, invasion rates and $A E G-1$ expression, and that overexpressed AEG-1 demonstrated its carcinogenesis, which resulted in increased proliferation, migration and invasion of the tumor cells and the 
A
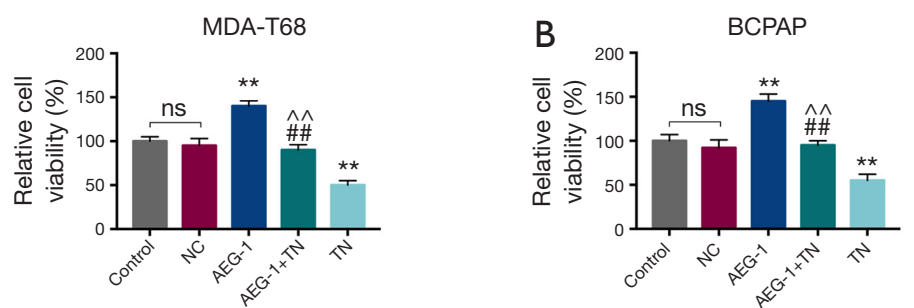

C

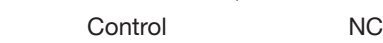

NC AEG-1

AEG-1+TN

MDA-T68
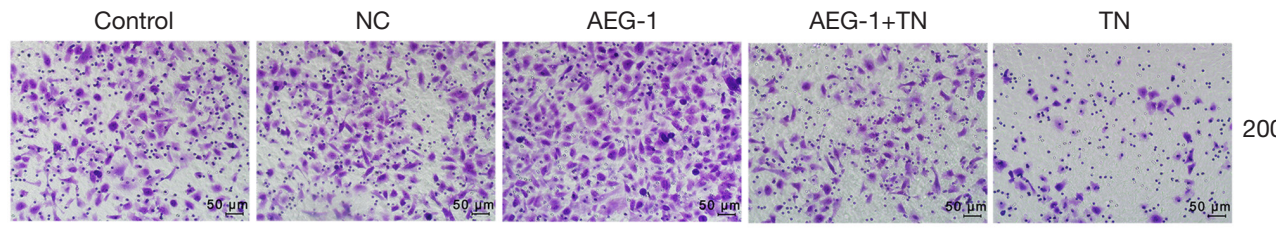

$200 \times$

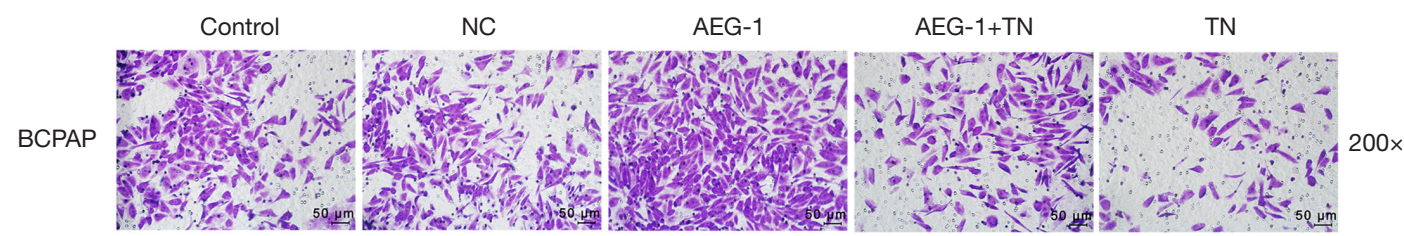

D

MDA-T68

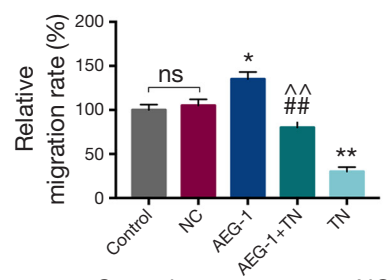

E

$\mathrm{F}$

Control

NC

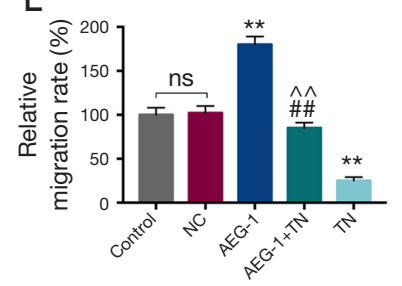
AEG-1
AEG-1+TN
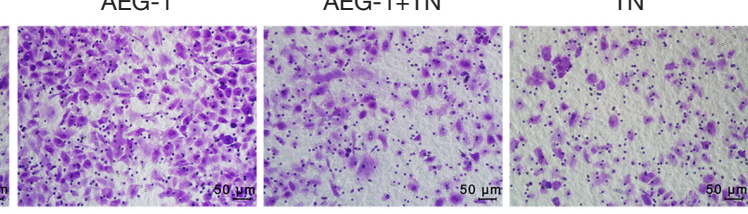

$200 \times$

MDA-T68

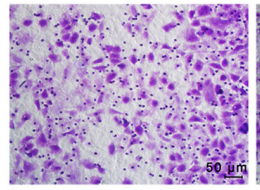

Control

NC

AEG-1

AEG-1+TN

TN
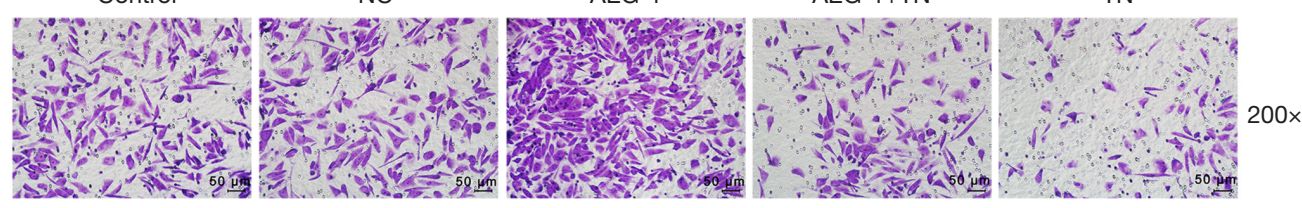

G
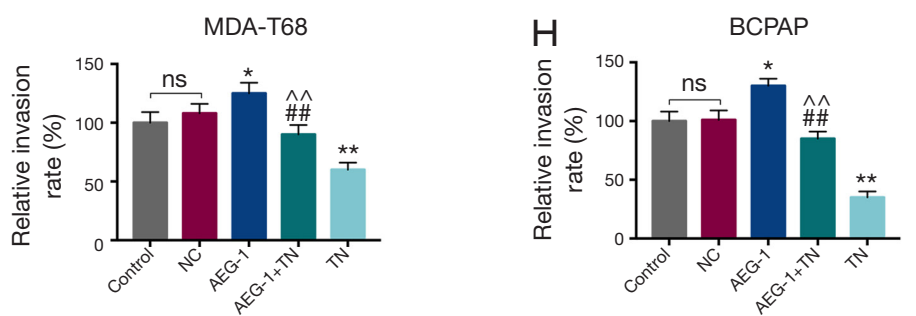

Figure 3 TN effects on metastasis of thyroid cancer cells with a relative high expression of AEG-1. (A) Relative cell viability in MDA-T68 and (B) BCPAP cells in Control, NC, AEG-1, AEG-1 + TN and TN groups; (C) representative images showed the MDA-T68 and BCPAP cells migrated into the bottom chamber in the groups, the cells were stained by $0.2 \%$ crystal violet; (D) relative migration rate (\%) in MDA-T68 and (E) BCPAP cells in the groups; (F) representative images showed the MDA-T68 and BCPAP cells invaded into the bottom chamber in the groups, the cells were stained by $0.2 \%$ crystal violet; $(\mathrm{G})$ relative invasion rate (\%) in MDA-T68 and (H) BCPAP cells in the groups. Bars indicated mean $\pm \mathrm{SD} .{ }^{*} \mathrm{P}<0.05$ or ${ }^{* *} \mathrm{P}<0.001$ versus Control or $\mathrm{NC}$ group; ${ }^{\# \#} \mathrm{P}<0.001$ versus $\mathrm{AEG}-1$ group; ${ }^{\wedge} \mathrm{P}<0.001$ versus $\mathrm{TN}$ group. 
A

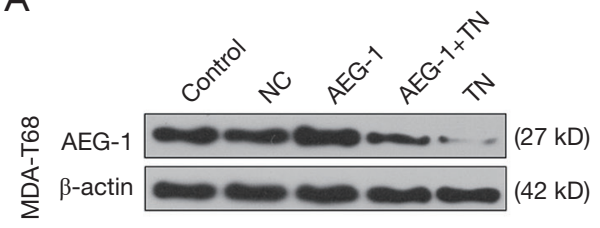

D

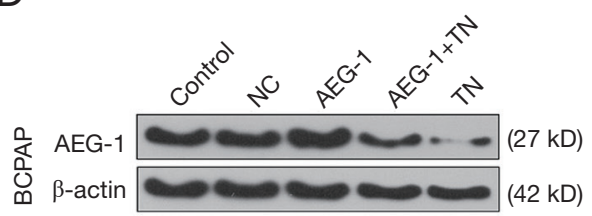

G
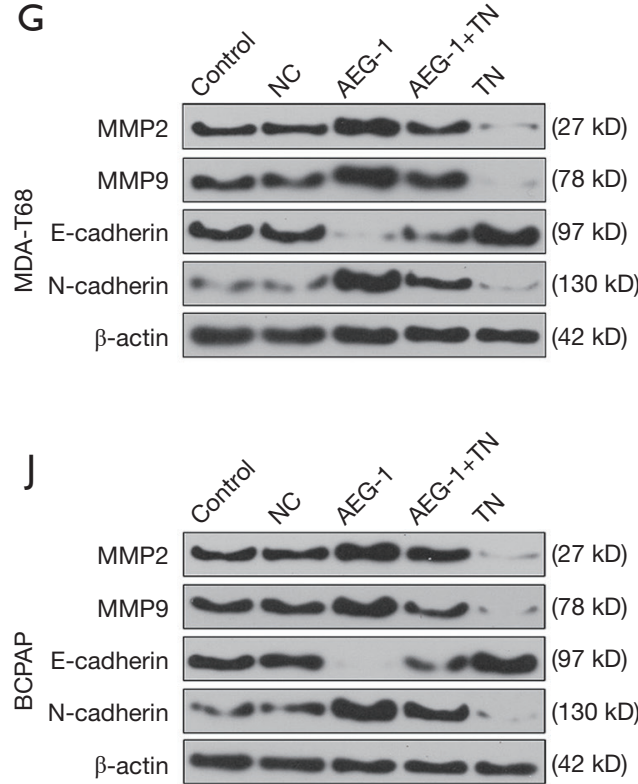

B MDA-T68

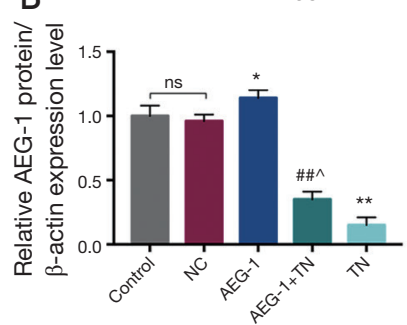

E

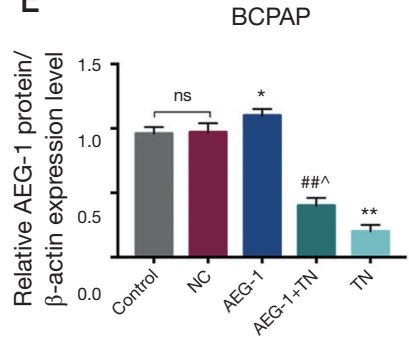

$\mathrm{H}$

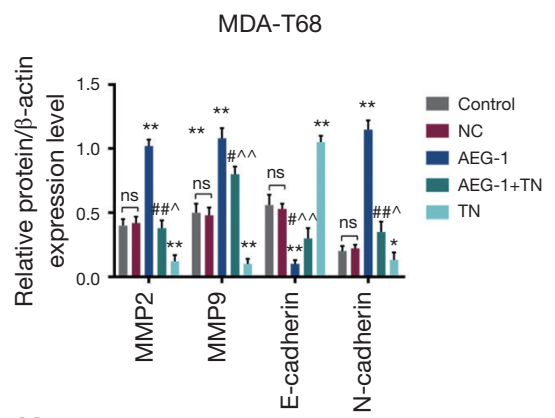

K

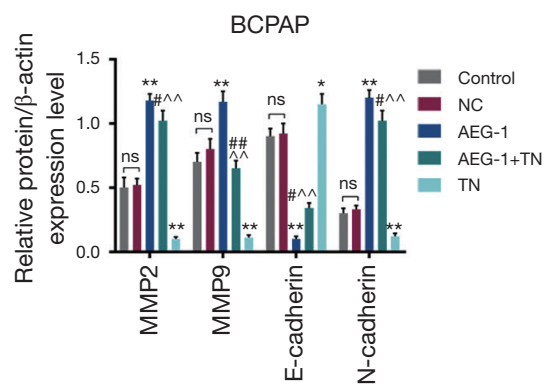

C

MDA-T68

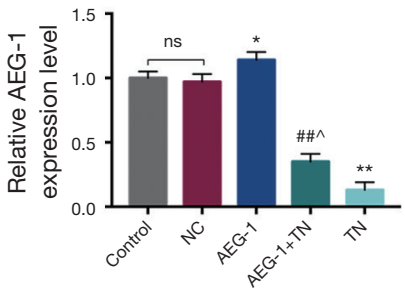

$\mathrm{F}$

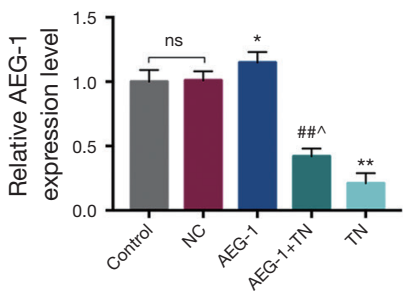

I

MDA-T68

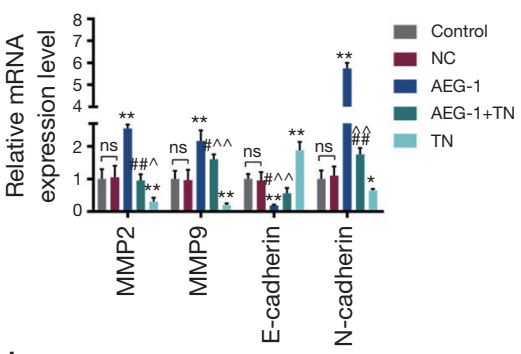

L

BCPAP

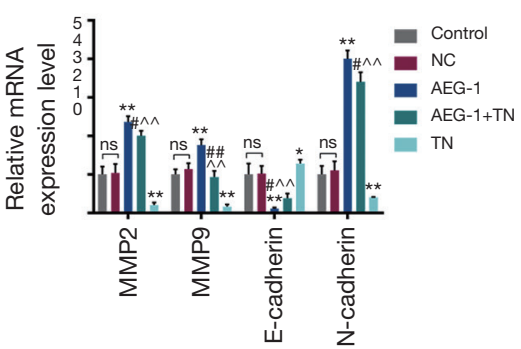

Figure 4 TN effects on AEG-1 expression and epithelial-mesenchymal transition (EMT)-related genes in thyroid cancer cells with a relatively high expression of AEG-1. (A) AEG-1 protein bolts from MDA-T68 cells by Western blot in Control, NC, AEG-1, AEG-1 + TN and TN group; (B) relative AEG-1 protein expression and (C) mRNA expression in MDA-T68 cells in the groups; (D) AEG-1 protein bolts from BCPAP cells by Western blot in the groups; (E) Relative AEG-1 protein expression and (F) mRNA expression in BCPAP cells in the groups; (G) protein expression of EMT-related genes from MDA-T68 cells in the groups; (H) Relative protein expression of EMT-related genes and (I) their mRNA expression levels in MDA-T68 cells in the groups; (J) protein expression of EMT-related genes from BCPAP cells in the groups; (K) relative protein expression of EMT-related genes and $(\mathrm{L})$ their mRNA expression levels in BCPAP cells in the groups. Bars indicated mean $\pm \mathrm{SD}$. ${ }^{*} \mathrm{P}<0.05$ or ${ }^{* *} \mathrm{P}<0.001$ versus Control or $\mathrm{NC}$ group; ${ }^{\#} \mathrm{P}<0.05$ or ${ }^{\# \#} \mathrm{P}<0.001$ versus $\mathrm{AEG}-1$ group; ${ }^{\wedge} \mathrm{P}<0.05$ or $\wedge \wedge \mathrm{P}<0.001$ versus $\mathrm{TN}$ group. 
expressions of MMP2, MMP9 and N-cadherin and decreased E-cadherin expression. However, TN could counteract such effects of overexpressed AEG-1. Thus, the present study showed the potential of TN in the treatment of thyroid cancer.

We found that TN negatively affects the proliferation, migration and invasion of thyroid cancer cells. Dong et al. revealed that $\mathrm{TN}$ inhibits prostate cancer cells through mammalian target of rapamycin (mTOR)-related signal pathway (8). Yang et al. demonstrated that TN could suppress the proliferation of human B-lymphoma Raji and T-lymphoma Jurkat cells (9). Furthermore, Wang et al. observed that the abilities of migration and invasion of gastric cancer-associated fibroblasts (GCAFs) could be greatly inhibited by TN (10). Thus, TN could be explored to inhibit the progression of cancers, including thyroid cancer.

The present study also discovered that $A E G-1$ overexpression increased the cell viability, migration and invasion of MDA-T68 and BCPAP cells, meanwhile, the expressions of MMP2, MMP9 and N-cadherin of thyroid cancer cell lines were significantly elevated by $A E G-1$ overexpression.

AEG-1, alternatively known as Metadherin (MTDH), can act as an important molecule in the regulation of multiple events in cancer development $(11,12)$. Studies have shown that $A E G-1$ expression is elevated in a variety of cancers and it has become a potential key regulator of malignancies mediated by oncogenic signaling pathways $(13,14)$. AEG-1 is involved in the proliferation of cancer cells and pathological processes, and also plays a key role in signaling pathways, such as the activation of phosphatidylinositol 3-kinase (PI3K)/AKT serine/threonine kinase (Akt) signaling (15), nuclear factor of kappa light polypeptide gene enhancer in B cells (NF-кB) (16), Wnt/ $\beta$-catenin (17) and mitogen-activated protein kinase (MAPK) pathway (18).

During EMT, epithelial cells lose their polarity and transform into a more active form that allows them to move easily between cell matrices under certain pathological conditions (19). EMT plays an important role in the differentiation of various tissues and organs, and metastasis of malignant tumor cells (20). EMT indicates a loss of epithelial phenotype marker E-Cadherin, and an increase of interstitial phenotype marker N-Cadherin (21). E-Cadherin is a calcium-dependent transmembrane glycoprotein that promotes cell adhesion and reduces cell migration (22).
$\mathrm{N}$-Cadherin belongs to the family of adhesion molecules and is also known as the marker of transmembrane glycoproteins and interstitial phenotypes (23). Studies demonstrated that $\mathrm{N}$-Cadherin interacts with the fiber growth factor receptor to promote the invasion of tumor cells (24).

MMPs are a class of zinc and calcium ion-dependent proteolytic enzymes that catalyze hydrolysis of extracellular matrices (25). MMP functions with tissue inhibitor of matrix metalloproteinase (TIMP) to regulate the degradation of extracellular matrix, and the imbalance of regulatory mechanisms can cause excessive degradation of extracellular matrix $(26,27)$. The degradation of extracellular matrix and basement membrane is critical for tumor metastasis, thus, the expression level of MMPs could reflect metastatic potential of tumors (28). Research revealed the intimate correlation between MMP2, MMP9 and thyroid cancer $(28,29)$. In addition, Huang et al. found the mechanism through which AEG-1 modulates the metastasis of papillary thyroid cancer via MMP2 and MMP9 (30). Fang et al. demonstrated that AEG-1 could be targeted by miR-625-3p, and further promoted the tumorigenesis of thyroid cancer (31). Taken together, AEG-1 was proved to be gene that could promote growth of thyroid cancer.

In the present study, the expression of AEG-1 in both MDA-T68 and BCPAP cells was greatly reduced by TN at the concentration of $50 \mathrm{nmol} / \mathrm{L}$. Moreover, the treatment of TN significantly inhibited the cell viability, migration, invasion rates and MMP2, MMP9, N-cadherin expressions of MDA-T68 and BCPAP cells, which, however, could be increased by AEG-1 overexpression. The results also showed that TN could increase E-cadherin expression, which could be decreased by AEG-1 overexpression. To the best of our knowledge, the present study is the first to show the relationship of TN and AEG-1 in thyroid cancer cells. Our results suggest that AEG-1 could be targeted and inhibited by $\mathrm{TN}$ in thyroid cancer, however, the specific mechanism underlying the effects of TN on AEG-1 should be further studied.

\section{Conclusions}

In summary, $\mathrm{TN}$ might inhibit the metastasis potential of thyroid cancer cells via restricting the expression of AEG-1. Our findings reveal a novel mechanism of $\mathrm{TN}$ in the treatment of thyroid cancer, the mechanism which should be further explored in the study of thyroid cancer. 


\section{Acknowledgments}

Funding: This work was supported by the Nanjing Foundation for the Development of Medical Science \& Technology [Grant Number YKK16212]; the Sixth Academic Experience Inheritance Project of National Famous TMC Doctors [Grant Number zmdzf (2017) 29]; the Nanjing Famous Traditional Chinese Medicine Studio [Grant Number Ningwei TCM \{2017\} 8].

\section{Footnote}

Conflicts of Interest: All authors have completed the ICMJE uniform disclosure form (available at http://dx.doi. org/10.21037/tcr.2019.12.94). The authors have no conflicts of interest to declare.

Ethical Statement: The authors are accountable for all aspects of the work in ensuring that questions related to the accuracy or integrity of any part of the work are appropriately investigated and resolved. No human and animals are involved in this research.

Open Access Statement: This is an Open Access article distributed in accordance with the Creative Commons Attribution-NonCommercial-NoDerivs 4.0 International License (CC BY-NC-ND 4.0), which permits the noncommercial replication and distribution of the article with the strict proviso that no changes or edits are made and the original work is properly cited (including links to both the formal publication through the relevant DOI and the license). See: https://creativecommons.org/licenses/by-nc-nd/4.0/.

\section{References}

1. Ciavardelli D, Bellomo M, Consalvo A, et al. Metabolic Alterations of Thyroid Cancer as Potential Therapeutic Targets. Biomed Res Int 2017;2017:2545031.

2. Applewhite MK, James BC, Kaplan SP, et al. Quality of Life in Thyroid Cancer is Similar to That of Other Cancers with Worse Survival. World J Surg 2016;40:551-61.

3. Tiedje V, Stuschke M, Weber F, et al. Anaplastic thyroid carcinoma: review of treatment protocols. Endocr Relat Cancer 2018;25:R153-61.

4. Cui J, Chen X, Su JC. [Advanced progress of main pharmacology activities of triptolide]. Zhongguo Zhong Yao Za Zhi 2017;42:2655-8.
5. Li XX, Du FY, Liu HX, et al. Investigation of the active components in Tripterygium wilfordii leading to its acute hepatotoxicty and nephrotoxicity. J Ethnopharmacol 2015;162:238-43.

6. Zhang M, Tan S, Yu D, et al. Triptonide inhibits lung cancer cell tumorigenicity by selectively attenuating the Shh-Gli1 signaling pathway. Toxicol Appl Pharmacol 2019;365:1-8.

7. Han H, Du L, Cao Z, et al. Triptonide potently suppresses pancreatic cancer cell-mediated vasculogenic mimicry by inhibiting expression of VE-cadherin and chemokine ligand 2 genes. Eur J Pharmacol 2018;818:593-603.

8. Dong F, Yang P, Wang R, et al. Triptonide acts as a novel antiprostate cancer agent mainly through inhibition of mTOR signaling pathway. Prostate 2019;79:1284-93.

9. Yang P, Dong F, Zhou Q. Triptonide acts as a novel potent anti-lymphoma agent with low toxicity mainly through inhibition of proto-oncogene Lyn transcription and suppression of Lyn signal pathway. Toxicol Lett 2017;278:9-17.

10. Wang Z, Ma D, Wang C, et al. Triptonide inhibits the pathological functions of gastric cancer-associated fibroblasts. Biomed Pharmacother 2017;96:757-67.

11. Emdad L, Das SK, Hu B, et al. AEG-1/MTDH/LYRIC: A Promiscuous Protein Partner Critical in Cancer, Obesity, and CNS Diseases. Adv Cancer Res 2016;131:97-132.

12. Vartak-Sharma N, Nooka S, Ghorpade A. Astrocyte elevated gene-1 (AEG-1) and the A(E)Ging HIV/AIDSHAND. Prog Neurobiol 2017;157:133-57.

13. Pan Y, Guo X, Yang Z, et al. AEG-1 activates Wnt/PCP signaling to promote metastasis in tongue squamous cell carcinoma. Oncotarget 2016;7:2093-104.

14. Wang Z, Cao CJ, Huang LL, et al. EFEMP1 promotes the migration and invasion of osteosarcoma via MMP-2 with induction by AEG-1 via NF-kappaB signaling pathway. Oncotarget 2015;6:14191-208.

15. Xie Y, Zhong DW. AEG-1 is associated with hypoxiainduced hepatocellular carcinoma chemoresistance via regulating PI3K/AKT/HIF-1alpha/MDR-1 pathway. EXCLI J 2016;15:745-57.

16. Yu X, Wang Y, Qiu H, et al. AEG-1 Contributes to Metastasis in Hypoxia-Related Ovarian Cancer by Modulating the HIF-1alpha/NF-kappaB/VEGF Pathway. Biomed Res Int 2018;2018:3145689.

17. He W, He S, Wang Z, et al. Astrocyte elevated gene1(AEG-1) induces epithelial-mesenchymal transition in lung cancer through activating Wnt/beta-catenin signaling. BMC Cancer. 2015;15:107. 
18. Chang Y, Li B, Xu X, et al. Lentivirus-Mediated Knockdown of Astrocyte Elevated Gene-1 Inhibits Growth and Induces Apoptosis through MAPK Pathways in Human Retinoblastoma Cells. PLoS One 2016;11:e0148763.

19. Ma J, Zhao J, Lu J, et al. Cadherin-12 enhances proliferation in colorectal cancer cells and increases progression by promoting EMT. Tumour Biol 2016;37:9077-88.

20. Chen T, You Y, Jiang H, et al. Epithelial-mesenchymal transition (EMT): A biological process in the development, stem cell differentiation, and tumorigenesis. J Cell Physiol 2017;232:3261-72.

21. Loh CY, Chai JY, Tang TF, et al. The E-Cadherin and $\mathrm{N}$-Cadherin Switch in Epithelial-to-Mesenchymal Transition: Signaling, Therapeutic Implications, and Challenges. Cells 2019;8. doi: 10.3390/cells8101118.

22. Cousin H. Cadherins function during the collective cell migration of Xenopus Cranial Neural Crest cells: revisiting the role of E-cadherin. Mech Dev 2017;148:79-88.

23. Li B, Shi H, Wang F, et al. Expression of E-, P-and $\mathrm{N}$-cadherin and its clinical significance in cervical squamous cell carcinoma and precancerous lesions. PLoS One 2016;11:e0155910.

24. Nguyen T, Mège RM. N-Cadherin and Fibroblast Growth Factor Receptors crosstalk in the control of developmental and cancer cell migrations. Eur J Cell Biol 2016;95:415-26.
25. Talmi-Frank D, Altboum Z, Solomonov I, et al. Extracellular Matrix Proteolysis by MT1-MMP Contributes to Influenza-Related Tissue Damage and Mortality. Cell Host Microbe 2016;20:458-70.

26. Karli S, Ayala-Haedo JA, Feuer WJ, et al. Effect of prostaglandin analogs on matrix metalloproteinases and tissue inhibitor of metalloproteinases in eyelid muscle specimens. Clin Ophthalmol 2018;12:2039-46.

27. Kübler A, Luna B, Larsson C, et al. Mycobacterium tuberculosis dysregulates MMP/TIMP balance to drive rapid cavitation and unrestrained bacterial proliferation. J Pathol 2015;235:431-44.

28. Banday MZ, Sameer AS, Mir AH, et al. Matrix metalloproteinase (MMP) -2, -7 and -9 promoter polymorphisms in colorectal cancer in ethnic Kashmiri population - A case-control study and a mini review. Gene 2016;589:81-9.

29. Kalhori V, Tornquist K. MMP2 and MMP9 participate in S1P-induced invasion of follicular ML-1 thyroid cancer cells. Mol Cell Endocrinol 2015;404:113-22.

30. Huang LL, Wang Z, Cao CJ, et al. AEG-1 associates with metastasis in papillary thyroid cancer through upregulation of MMP2/9. Int J Oncol 2017;51:812-22.

31. Fang L, Kong D, Xu W. MicroRNA-625-3p promotes the proliferation, migration and invasion of thyroid cancer cells by up-regulating astrocyte elevated gene 1 . Biomed Pharmacother 2018;102:203-11.
Cite this article as: Fu L, Niu X, Jin R, Xu F, Ding J, Zhang L, Huang Z. Triptonide inhibits metastasis potential of thyroid cancer cells via astrocyte elevated gene-1. Transl Cancer Res 2020;9(2):1195-1204. doi: 10.21037/tcr.2019.12.94 